Human and Animal Health

Vol.61: e18160122, 2018

http://dx.doi.org/10.1590/1678-4324-2018160122

ISSN 1678-4324 Online Edition
BRAZILIAN ARCHIVES OF BIOLOGY AND TECHNOLOGY

AN INTERNATIONAL JOURNAL

\title{
Solexa Profiling Identifies Differentially Expressed MiRNAs Between Sexually Immature and Mature Equine Testis
}

\author{
Liangjun $\mathrm{He}^{\mathbf{1}}$, Shiwei Wang ${ }^{2}$, Haifeng Deng ${ }^{3}$, Hong Dong ${ }^{1}$, Jingbo Chen ${ }^{4 *}$. \\ ${ }^{1}$ Shihezi University - College of Animal Science, Shihezi, Xinjiang, China; ${ }^{2}$ Tarim University - College of Animal \\ Science, Aral, Xinjiang, China; ${ }^{3}$ Zhaosu Horse Farm, Yili, Xinjiang, China; ${ }^{4}$ Xinjiang Academy of Animal Science - \\ Institute of Animal Science, Urumqi, Xinjiang, China .
}

\begin{abstract}
MicroRNAs (miRNAs) are a class of short non-coding RNAs identified as potent regulators of gene expression. Previous studies have suggested that miRNAs are involved in mammalian spermatogenesis. Stallion fertility is an important trait for the horse breeding industry, but stallion fertility traits are largely ignored in the industry. In this study, we generated expression profiles of miRNAs in foal (immature) and stallion (mature) testes using Solexa sequencing. We identified 438 known and homologous equine miRNAs and 199 novel miRNAs which were distributed among all the chromosomes. The two developmental stages showed significant differences in miRNA expression patterns. Our result expands the horse miRNA database and provided additional information on the stallion fertility and possible spermatogenesis regulation through specific miRNAs.
\end{abstract}

Key words: horse; miRNAs; solexa; testis

\footnotetext{
*Author for correspondence: chenjb126@126.com
} 


\section{INTRODUCTION}

MicroRNAs (miRNAs) are short, non-coding, endogenous RNAs, 19 to 25 nucleotides (nts) in length, which have been identified as potent regulators of gene expression through post-transcriptional gene silencing. The first miRNA identified, lin-4, was found in the nematode Caenorhabditis elegans by standard positional cloning of genetic loci and is involved in developmental timing [ $\left.{ }^{1}\right]$. Biosynthesis of miRNAs begins with the transcription of the primary miRNA (pri-miRNA) by RNA polymerase II. The primiRNA is recognized and cleaved by a microprocessor complex, Drosha and DGCR8, to produce a stem-loop RNA (pre-miRNA), which is exported from the nucleus by exportin 5 into the cytoplasm and is then cleaved by Dicer to yield a double-stranded RNA. Subsequently, the double-stranded RNA is separated into single strands, and generally one of the two strands is incorporated into the RISC complex, while the other strand is degraded $\left[{ }^{2}\right]$. Most commonly, the RISC-incorporated miRNA binds to the 3'UTR of the target mRNA by base-pairing and consequently induces either translational repression or mRNA degradation $\left[{ }^{3}\right]$.

Male fertility requires that there are large numbers of normal spermatozoa in the testis, formed though a complex process known as spermatogenesis. Spermatogenesis is a precisely synchronized process, which involves mitotic cell division and propagation of spermatogonial stem cells (SSCs), meiotic division, and subsequent processing in the seminiferous tubules. The division of type A spermatogonia provides both self-renewal of SSCs and type B spermatogonia, which differentiate and divide mitotically into primary spermatocytes. During meiosis, primary spermatocytes divide into two secondary spermatocytes and then produce four haploid round spermatids, which contain half the original number of chromosomes. Finally, haploid cells undergo a morphologic transformation known as spermiogenesis to develop into mature spermatozoa.

Many studies have suggested that miRNAs are involved in spermatogenesis. The deletion of the Dicer gene (encoding an enzyme required for miRNA biogenesis) in mouse primordial germ cell results in retarded spermatogenesis, which demonstrates that this miRNA is essential for primordial germ cell and spermatogonia proliferation [ $\left.{ }^{4}\right]$. miRNA are also important for the late stages of spermatogenesis. The knockout of Dicerl in mouse germ cells causes decreasing germ cell number in the seminiferous tubules, impaired transition from round to elongated spermatids and abnormal sperm motility $\left[{ }^{5}\right]$. Immature and mature testes have different miRNA expression profiles, and many miRNAs are stage-specifically expressed in spermatogenesis. Yan et al. identified sox 5 and sox 6 as presumed targets of $m i R-181 c$ and $r s b n 1$ as putative target of $m i R-355$, $m i R-181 c$ and $m i R-181 b\left[^{6}\right]$.

The first study on equine miRNA identified 407 novel horse miRNA genes corresponding to 354 mature miRNAs, using a comparative genomics approach $\left.{ }^{7}\right]$. Illumina Next Generation Sequencing technology was used to identify 292 known miRNAs and 329 novel miRNAs in horse skeletal muscle, colon and liver tissues [ $\left.{ }^{8}\right]$. In order to discover sperm-based biomarkers for stallion fertility, sperm and testis transcriptomes were compared using microarray and RNA-seq $\left[{ }^{9}\right]$. In that study, the researchers found 6761 transcripts in sperm and 11,112 in testis, including 82 sperm miRNAs. Despite these efforts, complete miRNA expression profiles and functional annotation of miRNAs during spermatogenesis have not been characterized in horse.

A horse aged one year or younger is called a foal. At approximately $1.5 \mathrm{yr}$ of age, stallions reach puberty. Spermatogenesis is completed at 2 to 3 years of age. At four years old, stallions are mature in terms of testicular weight, daily sperm production and 
Solexa Profiling Horse Testis miRNAs

Sertoli cell number $\left[{ }^{10}\right]$. Sub-fertility in stallions increases veterinary fees and management costs and ultimately diminishes the genetic contribution from prized stud horses $\left[{ }^{11}\right]$. Therefore, understanding the differential miRNA expression between mature and immature equine testes may lead to a new direction in the search for biomarkers for stallion fertility and treatments for stallion infertility.

In this study, we used Solexa deep sequencing technology to characterize and compare miRNA expression profiles between sexually mature and immature horse testis to discover miRNA biomarkers for stallion fertility. As a result, we identified 438 known and homologous equine miRNAs and 199 novel miRNAs. These results show that the two testicular developmental stages have significantly different miRNA expression patterns that can be used as biomarkers of testicular maturity.

\section{MATERIALS AND METHODS}

\section{Tissue Collection}

In order to ensure the maturation of the testes, three Kazakh stallions (5-10 years old), who lived with a herd of mares $(n>20)$ in pasture during the breeding season in previous year and the pregnancy rate of each mare herd was higher than $70 \%$, were selected. Samples from these stallions were used as mature testes. Additional histological examination of all mature testes all showed normal spermatogenesis. Additionally, three normal Kazakh foals (immature, 5-8 months old) were castrated, and samples were collected as immature testes. The samples were immediately snap frozen in liquid nitrogen and stored in $-80^{\circ} \mathrm{C}$.

\section{Small RNA Library Construction and Sequencing}

Total RNA was extracted from the testes of the immature and mature testes samples using Trizol reagent (Invitrogen, USA). Subsequently, the three immature and three mature samples were pooled, respectively, to construct foal and stallion small RNA libraries. RNA quality was evaluated by an Agilent 2100 Bioanalyzer. Polyacrylamide gel electrophoresis (PAGE) was used to isolate the fraction containing RNA species of 10-40nt in length, which were then ligated with 3'- and 5'-adaptors. The adaptor-ligated RNAs were converted into single-stranded cDNAs and amplified by RT-PCR. Finally, the purified cDNA was sequenced on a Genome Analyzer according to the manufacturer's instructions.

\section{Expression Estimation and Differential Expression Analysis of Mirna}

We removed the adaptor sequences of the raw reads and filtered out reads in which more than $30 \%$ of bases had a quality $<20$. Then, identical reads were collapsed using fastx_collapser, and the counts of each unique sequence were recorded. Cleaned and collapsed reads were aligned against the equine draft genome using the Short Oligonucleotide Analysis Package (SOAP) (http://soap.genomics.org.cn). Sequences with no more than one mismatch were retained for further analysis. Next, the sequences were annotated by aligning them against the sequences of small non-coding RNAs (rRNA, tRNA, snRNA, and snoRNA) databases: Rfam-11.0, rnammer-1.2, snoRNALBME, GenomictRNAdatabase.These annotated small non-coding RNAs were excluded from the miRNAs expression analyses. The remaining uniquely mapped reads were aligned with equine miRNA sequences present in miRBase 21.0 to identify known miRNA in the domestic horse, Equus caballus. They were also searched against the currently known human, mouse and rat mature miRNAs to find conserved miRNA 
homologs. We used DEGseq version 1.20.0 package in $\mathrm{R}$ 3.1.1. (http://bioinfo.au.tsinghua.edu.cn/software/degseg) and set the threshold $p$ value $<0.001$ under MARS (MA-plot-based method with random sampling model) method to define differentially expressed miRNAs.

\section{Mirna Target Gene Prediction and Functional Enrichment Analysis}

Given the fact many E. caballus miRNAs have not been experimentally validated, we choose to identify reliable miRNA target genes using the strategy of searching for homologous miRNAs with other species. Equine miRNA sequences were searched in BLAST to find homologous miRNAs in the human genome. Validated targets of these homologous miRNAs were downloaded from miRTarbase (http://mirtarbase.mbc.nctu.edu.tw/), targets were retained if the support type is not "weak". Gene Ontology (GO) analysis of the targets of differentially expressed miRNAs was conducted using the topGO package, and KEGG enrichment analysis was performed using the pathview package.

\section{Quantitative Real-Time Rt-Pcr to Validate Mirna Expression}

Quantitative real-time RT-PCR (qRT-PCR) was conducted to validate the miRNA expression changes, as previously described $\left[{ }^{12}\right]$. We selected nine miRNAs, including five up-regulated miRNAs (miR-34c, miR-449a, miR-34b-5p, miR-34a and miR-221) and four down-regulated miRNAs (miR-411, miR-199a-3p, miR-let-7a and miR-148a) and examined their expression changes in the foal and stallion testis samples. miRNAspecific stem-loop RT primers, miRNA-specific PCR forward primers and universal reverse primers were designed as previously described $\left[{ }^{13}\right]$ (Table S1). GAPDH was used as the internal control for miRNA detection $\left[{ }^{14,15}\right]$.

Table 1. Top 10 biological processes for miRNA target genes

\begin{tabular}{|c|c|c|c|c|c|}
\hline GO.ID & Term & Annotated & Observed & Expected & P-value \\
\hline GO:0008283 & cell proliferation & 1689 & 86 & 22.24 & $<1 \mathrm{e}-30$ \\
\hline GO:0042127 & regulation of cell proliferation & 1288 & 73 & 16.96 & $1.20 \mathrm{E}-28$ \\
\hline GO:0010033 & response to organic substance & 2220 & 93 & 29.24 & $1.90 \mathrm{E}-27$ \\
\hline GO:0048519 & $\begin{array}{l}\text { negative regulation of } \\
\text { biological processes }\end{array}$ & 3457 & 113 & 45.53 & $2.80 \mathrm{E}-25$ \\
\hline GO:0042221 & response to chemical & 3136 & 104 & 41.3 & $4.70 \mathrm{E}-23$ \\
\hline GO:0048523 & $\begin{array}{c}\text { negative regulation of cellular } \\
\text { process }\end{array}$ & 3154 & 104 & 41.54 & $7.50 \mathrm{E}-23$ \\
\hline GO:0071310 & $\begin{array}{c}\text { cellular response to organic } \\
\text { substance }\end{array}$ & 1643 & 74 & 21.64 & $8.10 \mathrm{E}-23$ \\
\hline GO:0070887 & $\begin{array}{l}\text { cellular response to chemical } \\
\text { stimulus }\end{array}$ & 2065 & 83 & 27.2 & $1.00 \mathrm{E}-22$ \\
\hline GO:0048856 & $\begin{array}{c}\text { anatomical structure } \\
\text { development }\end{array}$ & 4327 & 123 & 56.99 & $1.10 \mathrm{E}-22$ \\
\hline GO:0009719 & $\begin{array}{l}\text { response to endogenous } \\
\text { stimulus }\end{array}$ & 1240 & 64 & 16.33 & $1.50 \mathrm{E}-22$ \\
\hline
\end{tabular}

One microgram total RNA was reverse-transcribed into cDNA using reverse transcriptase (RT; Revert Aid ${ }^{\mathrm{TM}} \mathrm{M}-\mathrm{MuLV}$ RT) and miRNA-specific stem-loop RT primers (Table S1). The mix was incubated at $37^{\circ} \mathrm{C}$ for $15 \mathrm{~min}, 85^{\circ} \mathrm{C}$ for $5 \mathrm{~min}$ and then at $4^{\circ} \mathrm{C}$ using an Applied Biosystems 9700 Thermocycler. SYBR Green RT-PCR assays were conducted as follows: $20 \mu \mathrm{L}$ reaction mixtures containing $1 \mu \mathrm{L}$ cDNA $(1: 10$ dilution) were prepared and incubated at $95^{\circ} \mathrm{C}$ for $3 \mathrm{~min}$, followed by 40 cycles of $95^{\circ} \mathrm{C}$ for $15 \mathrm{~s}$ and $60^{\circ} \mathrm{C}$ for $40 \mathrm{~s}$, using LightCycler 480 Software Setup (Roche). Melting curve 
Solexa Profiling Horse Testis miRNAs

analysis and agarose gel electrophoresis were used to confirm the specific PCR products. The qPCR validation was carried out for three biological replicates. The $2^{-}$ $\Delta \Delta \mathrm{Ct}$ method was used to determine the expression level differences of the miRNAs for samples.

\section{RESULTS}

\section{Small RNA Composition of the Horse Testis Libraries}

We performed Solexa deep sequencing of two small RNA libraries derived from three pooled foal samples and three pooled stallion samples. We obtained 9,250,474 and $9,813,836$ raw reads, respectively, from each library. After quality filtering, we had $9,176,703$ clean reads in the foal library (Table S2) and 9,683,245 clean reads in the stallion library (Table S3). RNAs of 21-23 nts in length comprised $79.43 \%$ of all small RNAs in the foal library, while the stallion library showed a bimodal distribution with the major peak at 22-23 nts and a secondary peak at 25-27 nts (Fig 1).

Table 2. Top 10 molecular functions for miRNA target genes

\begin{tabular}{|c|c|c|c|c|c|}
\hline GO.ID & Term & Annotated & Observed & Expected & P-value \\
\hline GO:0005515 & protein binding & 8120 & 162 & 101.15 & $2.80 \mathrm{E}-20$ \\
\hline GO:0019901 & protein kinase binding & 394 & 28 & 4.91 & $7.80 \mathrm{E}-14$ \\
\hline GO:0019900 & kinase binding & 436 & 29 & 5.43 & $1.50 \mathrm{E}-13$ \\
\hline GO:0019899 & enzyme binding & 1206 & 48 & 15.02 & $2.70 \mathrm{E}-13$ \\
\hline GO:0043565 & $\begin{array}{c}\text { sequence-specific DNA } \\
\text { binding }\end{array}$ & 715 & 35 & 8.91 & $2.80 \mathrm{E}-12$ \\
\hline GO:0008134 & $\begin{array}{l}\text { transcription factor } \\
\text { binding }\end{array}$ & 461 & 28 & 5.74 & $3.60 \mathrm{E}-12$ \\
\hline GO:0005488 & binding & 12393 & 187 & 154.39 & $7.20 \mathrm{E}-12$ \\
\hline GO:0044212 & $\begin{array}{l}\text { transcription regulatory } \\
\text { region DNA binding }\end{array}$ & 377 & 25 & 4.7 & $8.70 \mathrm{E}-12$ \\
\hline GO:0000975 & $\begin{array}{c}\text { regulatory region DNA } \\
\text { binding }\end{array}$ & 384 & 25 & 4.78 & $1.30 \mathrm{E}-11$ \\
\hline GO:0001067 & $\begin{array}{c}\text { regulatory region nucleic } \\
\text { acid binding }\end{array}$ & 384 & 25 & 4.78 & $1.30 \mathrm{E}-11$ \\
\hline
\end{tabular}

Table 3. Top 10 enriched pathways for miRNA target genes

\begin{tabular}{ccccc}
\hline Pathways & Annotated & Significant & Expected & P-value \\
\hline Pathways in cancer & 326 & 40 & 6.83 & $3.60 \mathrm{E}-21$ \\
Cell cycle & 124 & 19 & 2.60 & $5.72 \mathrm{E}-12$ \\
Chronic myeloid leukemia & 73 & 15 & 1.53 & $1.45 \mathrm{E}-11$ \\
Bladder cancer & 42 & 12 & 0.88 & $2.71 \mathrm{E}-11$ \\
Pancreatic cancer & 70 & 14 & 1.47 & $1.07 \mathrm{E}-10$ \\
Small cell lung cancer & 85 & 15 & 1.78 & $1.46 \mathrm{E}-10$ \\
Melanoma & 71 & 13 & 1.49 & $1.65 \mathrm{E}-09$ \\
Colorectal cancer & 62 & 12 & 1.30 & $3.72 \mathrm{E}-09$ \\
p53 signaling pathway & 68 & 12 & 1.42 & $1.13 \mathrm{E}-08$ \\
Prostate cancer & 89 & 13 & 1.86 & $2.90 \mathrm{E}-08$ \\
\hline
\end{tabular}




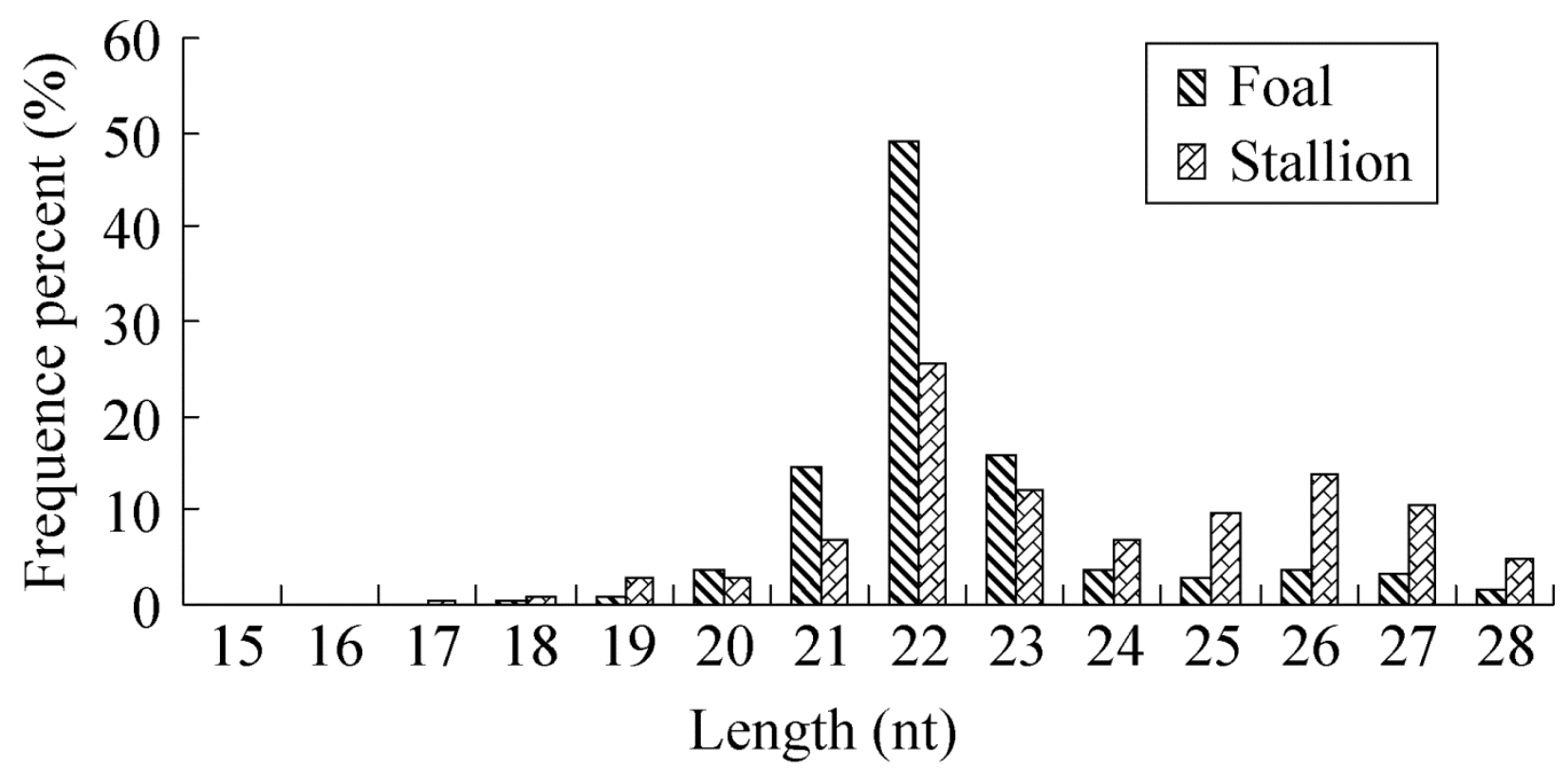

Figure1. Small RNA length distribution and abundance in libraries of foal and stallion

\section{Conserved Mirnas and Novel Mirnas}

The stallion library had a higher percent of unannotated small RNAs $(49.01 \%)$ than that of the foal $(25.75 \%)$ (Tables S2 and S3).

We detected 438 known and conserved miRNAs in our study (374 miRNAs in the foal library and 387 miRNAs in the stallion library) (Table S4). In total, 323 miRNAs were shared between the two libraries, but we also identified 135 novel candidate miRNAs (Table S5), of which 65 were found in the foals and 99 were found in the stallions.

We detected expression of nine members of the let-7 family, including let-7a, let-7a5p, let-7b-5p, let-7c, let-7d, let-7e, let-7f, let-7f-5p and let-7g. Let-7a, let-7f and let-7c were the most abundant miRNAs in the two libraries. Total read counts of the let-7 family were 4,864,334 and 2,546,959 in the foal and stallion libraries, respectively, which accounted for $82.67 \%$ and $83.45 \%$ of total known and conserved miRNA sequences in the corresponding libraries. This is consistent with a previous report that the members of the let-7 family are highly abundant and conserved miRNAs among both plants and animals $\left[{ }^{16}\right]$.

\section{Analysis and Validation of Differentially Expressed Mirnas}

We found 180 differentially expressed miRNAs between foal and stallion testes. Of these, 105 miRNAs were significantly down-regulated and 75 miRNAs were upregulated in stallion testes compared with foal testes (Figure 2 and Table S6).

To validate these expression changes, we used real-time qRT-PCR to determine the relative expression levels of nine randomly selected miRNAs (let-7a, miR-148a, miR199a-3p, miR-221, miR-34a, miR-34b-5p, miR-34c, miR-449a and miR-411). The validation results are shown in Figure 3, in which the x-axis represents the miRNAs and the $y$-axis is the $\log 2$ fold change between stallion and foal (log2 (stallion/foal) for both qRT-PCR and solexa sequencing). In general, the qRT-PCR results were consistent with the deep sequencing data. 
Solexa Profiling Horse Testis miRNAs

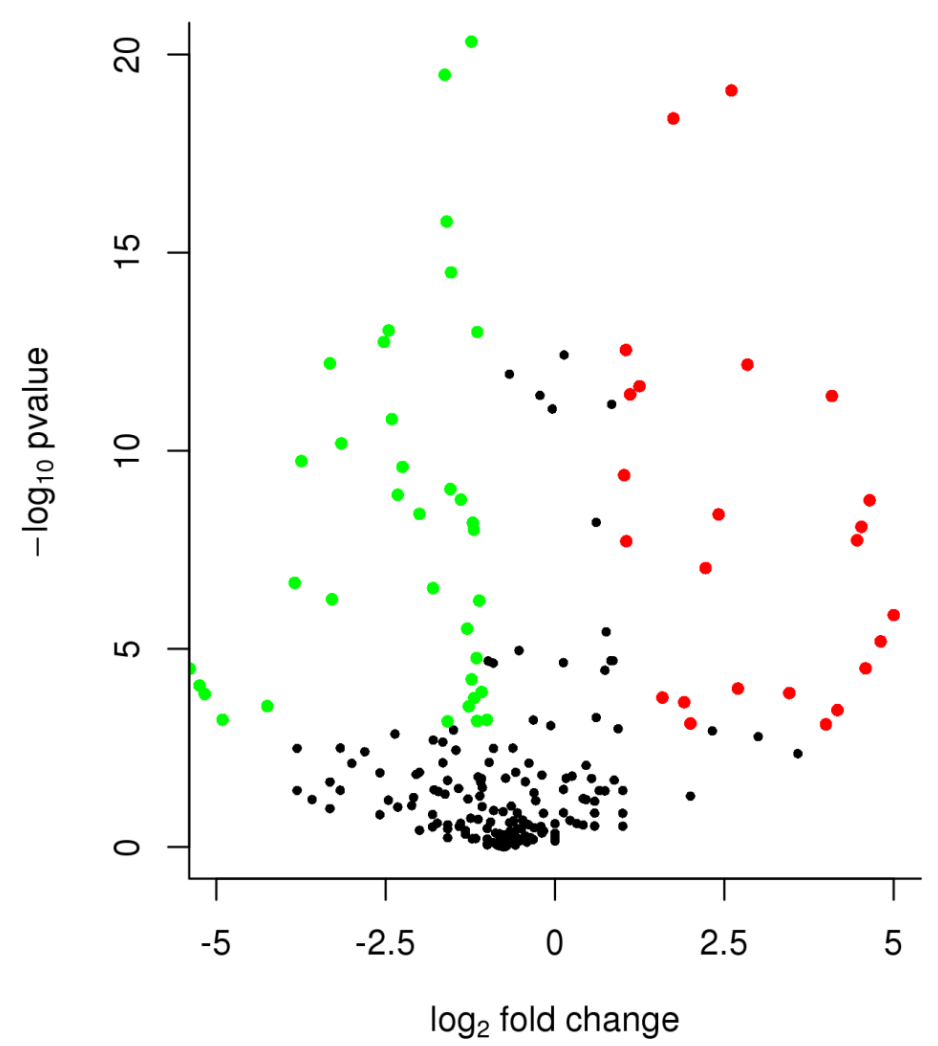

Figure 2. The volcano plot of differentially expressed miRNAs. The X-axis shows the $\log 2$-tran

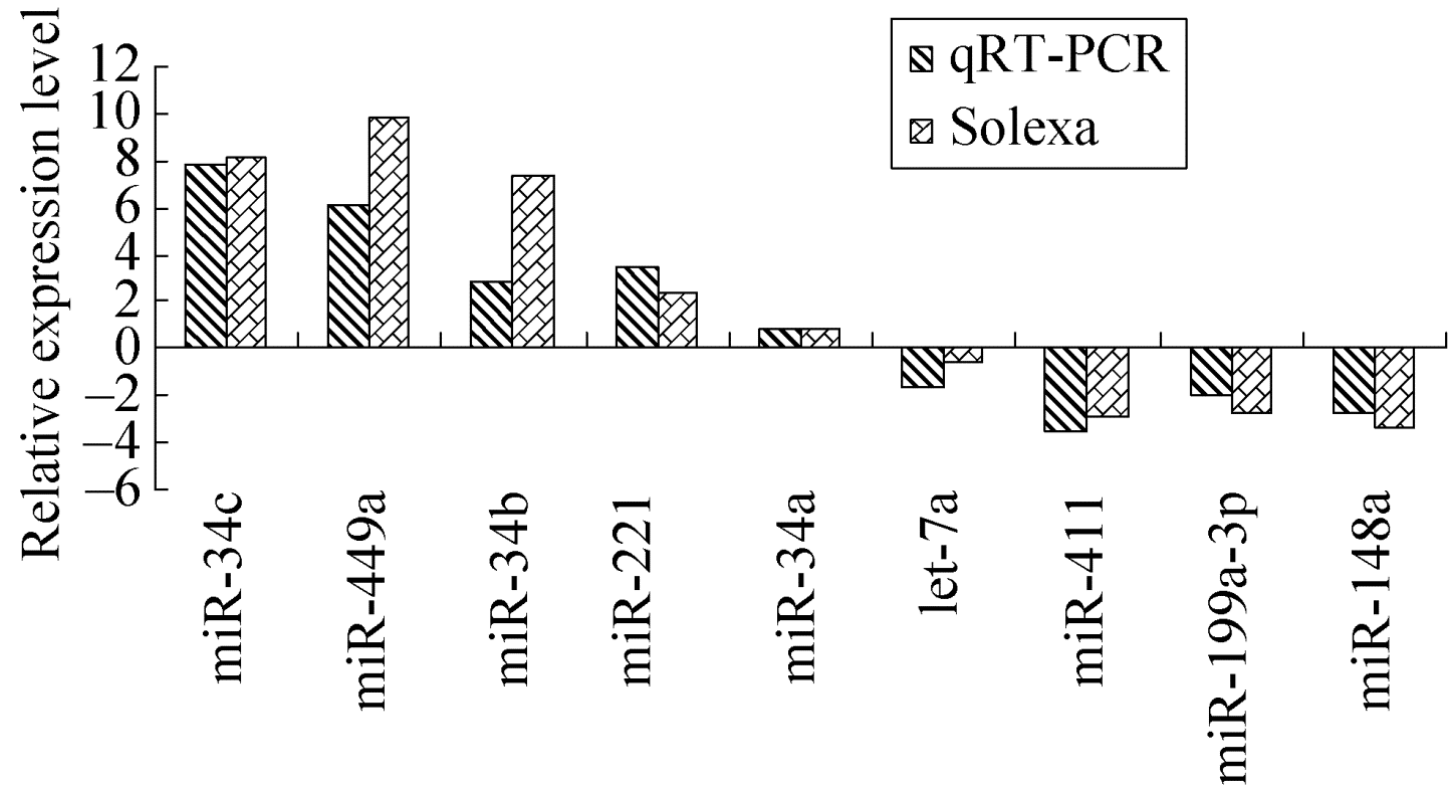

Figure 3. Validation of the sequencing results by qRT-PCR. The $x$-axis represents the miRN

\section{Mirnas Preferentially Expressed in Equine Testis}

A study $\left[{ }^{8}\right]$ measured the miRNA expression in horse skeletal muscle, colon and liver. Combining their data with the testicular miRNA expression data from the present study, 
we identified five preferentially expressed miRNAs in testis, which are eca-let-7a, ecalet-7f, eca-miR-432, eca-miR-503 and eca-miR-125a-5p. These miRNAs could be further used as biomarkers of horse testes.

\section{Functional Analysis of Differentially Expressed Mirnas}

We selected 15 abundant and differentially expressed miRNAs that had homologs in the human genome to find validated target genes and perform KEGG pathway analysis. As a result, 417 high confident validated miRNA-mRNA interactions were identified and further functional analysis are based on the validated genes (Table S8).

Interestingly, GO enrichment analysis showed that the target genes were highly involved in developmental and regulatory roles (Table 1 and Table 2). Two essential genes for self-renewal ( $S O X 2$ and $N A N O G$ ) and three important genes involved in apoptosis ( $M Y C, B C L 2$ and $B B C 3)$ were identified, which is consistent with results from mouse testes $\left[{ }^{6}\right]$.

Consistent with the predicted target genes of differentially expressed miRNAs between porcine mature and immature testes $\left[{ }^{17}\right]$, the KEGG pathway annotation also showed that the most enriched pathways were for cancer. This is not surprising considering the role of these miRNAs in development as seen in the GO analysis (Table S12).

\section{DISCUSSION}

The foal library has a length distribution peaked at $22 \mathrm{nt}$, a common length for miRNAs. The stallion library showed a bimodal distribution peaked at 21-23nt and at 25-27nt. Both agree with previous studies of immature and mature pig $\left[{ }^{17}\right]$ and human $\left[{ }^{18}\right]$ testis. According to studies of human RNAs, the second represents piRNAs, which are another class of small RNAs, mostly found in germ cells and required for spermatogenesis $\left[{ }^{19}\right]$. Since there is no known horse piRNA data bank, so many miRNA at the secondary peak didn't matched to piRNAs and was classified as unannotated small RNAs. It explains why the stallion library had higher percent of unannotated small RNAs than the foal library.

The difference in miRNA profiles between immature and mature testes has been studied in mouse $\left[{ }^{6}\right]$, pig $\left[{ }^{17}\right]$ and $\operatorname{dog}\left[{ }^{20}\right]$ using various techniques, including qRT-PCR, microarrays and Solexa sequencing. We compared our results with the miRNA expression seen in mouse and pig testes. In a study comparing miRNA expression between sexually mature and immature porcine testes, 96 significantly up-regulated miRNAs and 26 significantly down-regulated miRNAs were identified in the sexually mature porcine testes. We found six overlapping up-regulated miRNAs (miR-449a, $m i R-449 b, m i R-34 c-5 p, m i R-34 c, m i R-34 b-3 p, m i R-34 b-5 p$ and $m i R-184)$ and three overlapping down-regulated miRNAs (miR-411, miR-487b and miR-485-5p) between porcine and equine mature testes. Interestingly, five of the six overlapping up-regulated miRNAs were among the top ten up-regulated miRNAs and belong to two miRNA families, the $m i R-34$ family and the $m i R-449$ family. However, the overlapping downregulated miRNAs showed no specific pattern among the fold-change ranking. Using a microarray approach, another study compared the miRNA expression between immature and mature murine testes $\left[{ }^{6}\right]$ and revealed that $m i R-34 b-5 p, m i R-34 c-5 p$ and $m i R-449$ were up-regulated in mature murine testis, which is consistent with our results. It is noteworthy that $m i R-411$ was found to be significantly down-regulated in murine, porcine and equine mature testis, which suggests that $m i R-411$ might have a conserved function in testicular development in mammalian species. 
Solexa Profiling Horse Testis miRNAs

In these studies, the $m i R-34 b, m i R-34 c$ and $m i R-449$ families were the top up-regulated families in sexually mature testis. In the present study, $m i R-34 b-3 p, m i R-34 b-5 p, m i R$ $34 c, m i R-449 a$ and $m i R-449 b$ were the most up-regulated miRNAs in stallion testis. One previous study has reported that $m i R-449$ was preferentially expressed in murine testis, with the highest levels in spermatocytes and spermatids $\left[{ }^{21}\right]$. In mouse testis, miR-34c was expressed at very low levels before p12 (the prepubertal period), after which its expression increased sharply and persisted until adulthood in the mouse. Furthermore, this miRNA was localized in pachytene spermatocytes and was highly expressed in spermatids $\left[{ }^{22}\right]$. Bouhallier $\left[{ }^{23}\right]$ found that miR-34c highly expressed in pachytene spermatocytes and round spermatids, indicating that miR-34c was meiosis-specific. In human seminal plasma, miR-34c-5p was markedly decreased in azoopermia and increased in asthenopermia $\left[{ }^{24}\right]$. Additionally, miR-34b-3p, miR-34b-5p and miR-34c$5 p$ were found to be significantly decreased in subfertile and nonobstructive azoopermatic males. Administration of a CYP26B1 inhibiter, which intervened in retinoic acid-mediated spermatogenesis, resulted in significantly up-regulated $m i R-34 b$ and $m i R-34 c-5 p$ in cultured canine testicular parenchyma $\left[{ }^{25}\right] . M i R-34 b, m i R-34 c$ and $m i R-449$ family are similar in nucleotide sequence and have identical seed sequence $\left[{ }^{21}\right]$. In mouse testis, miR-449 deletion up-regulated miR-34b and $m i R-34 c$ expression but mouse spermatogenesis is not affected, which suggested miR-34b, miR-34c and miR449 family is functionally redundant in murine testis $\left[{ }^{21}\right]$.

Among the predicted miRNA target genes, CDK4, CCNE2, BCL2, and NOTCH1 were targeted by multiple differentially expressed miRNAs. CDK4 is an important regulator for the G1 to S phase cell cycle transition. The majority of $C d k 4^{-/}$male mice displayed infertility $\left[{ }^{26}\right]$, and $C d k 4^{--}$male mice showed age-dependent testes block in spermatogenesis $\left[{ }^{27}\right]$. CCNE2 is a G1 cyclin that associates with Cdk2 and is required for driving cells to enter the $\mathrm{S}$ phase of the cell cycle and for cell proliferation. CCNE2- male mice displayed reduced fertility, testicular size and sperm count and frequent abnormal meiosis in spermatocytes, suggesting that CCNE2 is essential for normal spermatogenesis $\left[{ }^{28}\right]$. BCL2 is a member of the Bcl-2 family, which regulates apoptosis. $\mathrm{Bcl}-2$ has been shown to be targeted by has-miR-34b, has-miR-34c and has-miR-449a. Yamamoto reported that overexpression of $\mathrm{Bcl}-2$ in transgenic mice testis impaired spermatogenesis $\left[{ }^{29}\right]$. The expression of exogenous $\mathrm{Bcl}-2$ in spermatogonia resulted in abnormal accumulation of spermatogonia and degeneration of germ cells, indicating that apoptosis is essential for normal spermatogenesis $\left[{ }^{30}\right]$. NOTCHI is one of the validated target genes for has-miR-34b, has-miR-34c and has-miR-449a in miRTar base. It has been reported that NOTCH1 is critical for germ cell development and differentiation in rat testis, and NOTCH1 is not detected in the testes of patients with spermatogenic maturation arrest $\left[{ }^{31}\right]$. In summary, the GO term and KEGG pathway annotation for differentially expressed miRNAs suggests likely roles for these miRNAs in spermatogenesis.

In this study, there were 77 miRNAs derived from genes on the $\mathrm{X}$ chromosome, of which 29 were up-regulated and 18 were down-regulated in the stallion compared to the foal. Using Fisher's test, we observed that significantly more differentially expressed miRNA is present on the $\mathrm{X}$ chromosome $(P=0.0051)$.

It is worth noting that the equine miR-8908 family, which consists of 14 members, all are clustered on the $\mathrm{X}$ chromosome except miR-8908n. Intriguingly, 10 of the $14 \mathrm{miR}$ 8908 family members were up-regulated in sexually mature equine testes, and the miR8908 family accounted for $13 \%$ (10/75) of all up-regulated miRNAs. Most members of the $m i R-8908$ family were up-regulated in mature equine testes and they accounted for 
a considerable percent of all up-regulated miRNAs in that sample, which suggests that the miR-8908 family may be essential for equine spermatogenesis.

Besides the 10 up-regulated $m i R$ - 8908 family members, there were 29 up-regulated Xlinked miRNAs, which accounted for $39 \%$ of all up-regulated miRNAs (29/75) in stallion testis, a significantly high ratio compared to the whole genome. It has been reported that some miRNAs from the $\mathrm{X}$ chromosome escape meiotic sex chromosome inactivation during spermatogenesis $\left[{ }^{32}\right]$. Those miRNAs were found in spermatocytes from the mid- to late pachytene stage of spermatogenesis. Our finding of a high percentage of up-regulated X-linked miRNAs is consistent with this report.

\section{CONCLUSIONS}

We compared the expression profiles of miRNAs in foal (immature) and stallion (mature) testes using Solexa sequencing. These differentially expressed miRNAs will help us to better understand the mechanisms involved in spermatogenesis and may lay the foundation for research into stallion infertility.

\section{REFERENCES}

1. Lee RC, Feinbaum RL, Ambros V. The C. elegans heterochronic gene lin-4 encodes small RNAs with antisense complementarity to lin-14. Cell. 1993;75:843-54.

2. Lee Y, Ahn C, Han J, Choi H, Kim J, Yim J, et al.The nuclear RNase III drosha initiates microRNA processing. Nature. 2003; 425:415-9.

3. Kim VN. MicroRNA biogenesis: coordinated cropping and dicing. Nat Rev Mol Cell Biol. 2005; 6: 376-85.

4. Hayashi K, Sousa-Lopes SMC, Kaneda M, Tang F, Hajkova P, Lao K, et al. MicroRNA biogenesis is required for mouse primordial germ cell development and spermatogenesis. PLoS One. 2008; 3: e1738.

5. Maatouk DM, Loveland KL, McManus MT, Moore K, Harfe BD. Dicer is required for differentiation of the mouse male germline. Biol Reprod. 2008;79:696-703.

6. Yan NH, Lu YL, Sun HQ, Tao DC, Zhang SZ, Liu WY, et al. A microarray for microRNA profiling in mouse testistissues. Reproduction. 2007; 134: 73-9.

7. Zhou M. In silico detection and characteristics of novel microRNA genes in the Equus caballus genome using anintegrated ab initio and comparative genomic approach. Genomics. 2009; 94:125-31.

8. Kim MC, Lee SW, Ryu DY, Cui FJ, Bhak J, Kimc Y. Identification and characterization of microRNAs in normal Equine tissues by next generation sequencing. PLoS ONE. 2014; 9:e93662.

9. Das PJ, Mccarthy F, Vishnoi M, Paria N, Gresham C, Li G, et al. Stallion sperm transcriptome comprises functionally coherent coding and regulatory RNAs as revealed by microarray analysis and RNA-seq. PLoS ONE. 2013;8:e56535.

10. Johnson L, Varner DD, Thompson DL Jr. Effect of age and season on the establishment of spermatogenesis in the horse. J Reprod Fertil. 1991; 44(Suppl):87-97.

11. Turner R. Oristaglio. Pathogenesis, diagnosis, and management of testicular degeneration in stallions. Clin TechEquine Pract. 2007; 6:278-84.

12. Varkonyi-Gasic E, Wu R, Wood M, Walton EF, Hellens RP. Protocol: a highly sensitive RTPCR method for detection and quantification of microRNAs. Plant Methods. 2007; 3:12.

13. Chen C, Ridzon DA, Broomer AJ, Zhou Z, Lee DH, J. Nguyen T, et al. Real-time quantification of microRNAs by stem-loop RT-PCR. Nucleic Acids Res. 2005; 33:e179.

14. Ing N H, Laughlin A M, Varner D D, et al. Gene expression in the spermatogenically inactive "Dark" and maturing "Light" testicular tissues of the prepubertal colt. J Androl. 2004, 24:535-44. 
15. Shields J E, Kochan K J, Jeong J, et al. Initial characterization of a gene abundantly expressed in stallion testis. J Equine Vet Sci, 2009, 29(5): 324-5.

16. Rouch S, Slack FJ.The let-7 family of microRNAs. Trends Cell Biol. 2008; 18: 505-516.

17. Lian CJ, Sun BX, Niu SL, Yang RJ, Liu BY, Lu CY, et al. A comparative profile of the microRNA transcriptome in immature and mature porcine testes using solexa deep sequencing. FEBS Joural. 2012;279:964-75.

18. Yang QL, Hua J, Wang L, Xu B, Zhang H, Ye N, et al. MicroRNA and piRNA profiles in normal human testis detected by next generation sequencing. Plos ONE.2013; 6:e66809.

19. Kim VN. Small RNAs just got bigger: piwi-interacting RNAs (piRNAs) in mammalian testes. Gene Dev. 2006; 20:1993-1997.

20. Kasimanickam V R, Kasimanickam RK. Differential expression of microRNAs in sexually immature and mature canine testes. Theriogenology. 2015; 83:394-8.

21. Bao JQ, Li D, Wang L, Wu JW, Hu YQ, Wang ZG, et al. MicroRNA-449 and MicroRNA34b/c Function Redundantly in Murine Testes by Targeting E2F Transcription FactorRetinoblastoma Protein (E2F-pRb) Pathway. J Biol Chem. 2012; 287: 21686-98.

22. Liang X, Zhou D, Wei C, Luo H, Liu J, Fu R, et al. MicroRNA-34c enhances murine male germ cell apoptosis through targeting ATF1. PLoS ONE. 2012; 7: e33861.

23. Bouhallier F, Allioli N, Lavial F. Role of miR-34c microRNA in the late steps of spermatogenesis. RNA. 2010; 16:720-731.

24. Abu-Halima M, Hammadeh M, Schmitt J, Leidinger P, Keller A, Meese E, et al. Altered microRNA expression profiles of human spermatozoa in patients with different spermatogenic impairments. Fertil Steril. 2013; 99:1249-55.

25. Kasimanickam VR, Kasimanickam RK. Dysregulated microRNA clusters in response to retinoic acid and CYP26B1 inhibitor induced testicular function in dogs. PLoS ONE. 2014; 9:e99433.

26. Rane SG, Dubus P, Mettus RV, Galbreath EJ, Boden G, Reddy EP, et al. Loss of Cdk4 expression causes insulin-deficient diabetes and $\mathrm{Cdk} 4$ activation results in beta-islet cell hyperplasia. Nat Genet. 1999; 22:44-52.

27. Mettus RV, Rane SG. Characterization of the abnormal pancreatic development, reduced growth and infertility in Cdk4 mutant mice. Oncogene. 2003; 22:8413-21.

28. Geng Y, Yu Q, Sicinska E, Das M, Schneider JE, Bhattacharya S, et al. Cyclin e ablation in the mouse. Cell. 2003; 114:431-43.

29. Yamamoto CM, Hikim AP, Lue Y, Portugal AM, Guo TB, Hsu SY, et al. Impairment of spermatogenesis in transgenic mice with selective overexpression of Bcl-2 in the somatic cells of the testis. J Androl. 2001; 22: 981-91.

30. Furuchi T, Masuko K, Nishimune Y, Obinata M, Matsui Y. Inhibition of testicular germ cell apoptosis and differentiation in mice misexpressing Bcl-2 in spermatogonia. Development. 1996; 122:1703-9.

31. Hayashi T, Kageyama Y, Ishizaka K, Xia G, Kihara K, Oshima H. Requirement of Notch 1 and its ligand jagged 2 expressions for spermatogenesis in rat and human testes. $\mathbf{J}$ Androl. 2001; 22:999-1011.

32. Song R, Ro S, Michaels JD, Park C, McCarrey JR. Many X-linked microRNAs escape meiotic sex chromosome inactivation. Nat Genet. 2009; 41:488 - 93.

Received: February 26, 2016; Accepted: August 02, 2016 
Table s5 Novel miRNAs Detected in Foal and Stallion Testis

\begin{tabular}{|l|l|l|}
\hline Novel miRnA of Foal & Count & Sequence \\
\hline >novel_mir_42 & 816371 & TGAGGTAGTAGGTTGTGTGGTT \\
\hline >novel_mir_48 & 207169 & TACCACAGGGTAGAACCACGGAC \\
\hline >novel_mir_63 & 43082 & TGAGGTAGTAGATTGTATAGTTT \\
\hline >novel_mir_60 & 1597 & AGGTCCCTGTTCGGGCGCCA \\
\hline >novel_mir_32 & 711 & TGAGGTAGTAGGTTGTATAGTTTT \\
\hline >novel_mir_28 & 582 & ACGATGATGATGGTGGTGAAG \\
\hline >novel_mir_65 & 437 & AGAGGTAAAAAATTGATTTGACT \\
\hline >novel_mir_5 & 306 & CAGTGCAATGTTAAAAGGGCATT \\
\hline >novel_mir_14 & 256 & CCGCACTGTGGGTACTTGCTGC \\
\hline >novel_mir_36 & 153 & CACAATACACGGTCGACCTCT \\
\hline >novel_mir_22 & 143 & ACTGCAGTGAAGGCACTTGTAG \\
\hline >novel_mir_23 & 60 & TATTGCACTTGTCCCGGCCTGTT \\
\hline >novel_mir_62 & 46 & CTGGGAGGTGGATGTTTACTT \\
\hline >novel_mir_64 & 46 & TACGTAGATATATATGTATTTT \\
\hline >novel_mir_45 & 41 & TGAGTGTGTGTGTGTGAGTGA \\
\hline >novel_mir_39 & 34 & CGGGGCAGCTCAGTACAGGATG \\
\hline >novel_mir_54 & 33 & TTGTTGGAGGGAATATACATAT \\
\hline >novel_mir_58 & 32 & TAGGTAGTTTCATGTTGTTGGGA \\
\hline >novel_mir_35 & 28 & ATGTCACTCGGCCCACTACCC \\
\hline >novel_mir_34 & 27 & ATCATAGAGGAAAATCCACAT \\
\hline >novel_mir_7 & 25 & TGCTGGGACCCGAAGCGCGACGGC \\
\hline >novel_mir_15 & 23 & TGCACTGTCAGAAGCGTATCCCTT \\
\hline >novel_mir_29 & 22 & TCTTTGCAGGCTTGAACAAGGGTT \\
\hline >novel_mir_21 & 20 & TACTGTTGTGAGTGGATCGTCTCT \\
\hline >novel_mir_57 & 18 & AAGGGAAAGGTGGGTCAAGGA \\
\hline >novel_mir_30 & 15 & TCAAGGGCCAGAGAACGCAGGAGA \\
\hline >novel_mir_51 & 15 & AAATTACAGATTGTCTAAGAGG \\
\hline >novel_mir_24 & 14 & ACACCACTGGACCGGCCCGTT \\
\hline >novel_mir_43 & 14 & ACAGCTGTGAAATGGGCATGT \\
\hline >novel_mir_9 & 14 & TGGCTGTTGAGAAGACTCCCAGA \\
\hline >novel_mir_17 & 13 & CTGCCCTGGCCCGAGGGACCGAC \\
\hline >novel_mir_46 & 12 & TTGCTCAAGGGGAAGATGTAGGCA \\
\hline >novel_mir_47 & 12 & ATGGAACTTCAGACAACAGGGCT \\
\hline >novel_mir_38 & 11 & TGAGACTGAATGCTGAGCGCAGGA \\
\hline >novel_mir_61 & 11 & AAGGGGTGAGGAAGACATAGTGG \\
\hline >novel_mir_12 & ACGCACTGAAGGACCTCCCGGTAA \\
\hline >novel_mir_31 & CTGAATCAAAGTAGAGGGACTCCG \\
\hline >novel_mir_56 & 10 & CCGATACGGATGGCTGACTGTT \\
\hline >novel_mir_59 & & \\
\hline >novel_mir_8 & CAAGCTTGTGTCTATAGGTATG \\
\hline novel_mir_1 & & \\
\hline
\end{tabular}


Solexa Profiling Horse Testis miRNAs

\begin{tabular}{|l|l|l|}
\hline >novel_mir_11 & 8 & TGGAGGCAGCGAGGAGCACGT \\
\hline >novel_mir_16 & 8 & TCAGGCTCAGTCCCCTCCCGAT \\
\hline >novel_mir_20 & 8 & TATATATATATATGTACGTAT \\
\hline >novel_mir_52 & 8 & TACAGTACTGTGATAACTGAAGGA \\
\hline >novel_mir_18 & 7 & AGGGGAGAAGGTAAGCAGAGA \\
\hline >novel_mir_19 & 7 & ACTAGGACAAGGAAGCTGGCAG \\
\hline >novel_mir_33 & 7 & CACAGAACTTTTAACCAGTAGGCC \\
\hline >novel_mir_37 & 7 & TGATGAACTTATGAGGAGCTGCTA \\
\hline >novel_mir_44 & 7 & CAGGAGGAAGACTGAGGTGGAA \\
\hline >novel_mir_53 & 7 & TCTCAGTATAGAATTCCGCGGGC \\
\hline >novel_mir_55 & 7 & CGTGGTGCGGGACGGAGCGGA \\
\hline >novel_mir_25 & 6 & TCAATGTCTGTGAGTCGGCAGCTA \\
\hline >novel_mir_26 & 6 & ACTGACTGGGAATGAAAGGCTG \\
\hline >novel_mir_27 & 6 & TCACGCGGTGAGAGGAAGGACC \\
\hline >novel_mir_3 & 6 & ACCTTGGCTCTAGACTGCTTACT \\
\hline >novel_mir_4 & 6 & TGATAGAATGTTTGCACCAGTGAC \\
\hline >novel_mir_49 & 6 & ACCCGGGCTCTGTGGGCAGGCGG \\
\hline >novel_mir_10 & 5 & AGGAATTGCAGGGGCATCTTTATC \\
\hline >novel_mir_13 & 5 & TTGGCTCTGTGAGGTCGGCTCA \\
\hline >novel_mir_2 & 5 & TCGGAGTCAGGAACGGCGTCTGGC \\
\hline >novel_mir_40 & 5 & AAAGTTGGCAGATGGTGAGTGAA \\
\hline >novel_mir_41 & 5 & AGGAGTCTTGTTTCTACTTT \\
\hline >novel_mir_50 & 5 & TCCTTGATCTGGGTTGGCTGAG \\
\hline >novel_mir_6 & 5 & TCTGTGTAGATGACATCGTGTTC \\
\hline
\end{tabular}




\begin{tabular}{|c|c|c|}
\hline Novel miRNA of Stallion & Count & Sequence \\
\hline >novel_mir_42 & 582547 & TGAGGTAGTAGGTTGTGTGGTT \\
\hline >novel_mir_48 & 66380 & TACCACAGGGTAGAACCACGGAC \\
\hline >novel_mir_63 & 33011 & TGAGGTAGTAGATTGTATAGTTT \\
\hline >novel_mir_32 & 2420 & TGAGGTAGTAGGTTGTATAGTTTT \\
\hline >novel_mir_60 & 1951 & AGGTCCCTGTTCGGGCGCCA \\
\hline >novel_mir_36 & 572 & CACAATACACGGTCGACCTCT \\
\hline >novel_mir_28 & 541 & ACGATGATGATGGTGGTGAAG \\
\hline >novel_mir_65 & 219 & AGAGGTAAAAAATTGATTTGACT \\
\hline >novel_mir_1 & 123 & TCCGCTTCTCGGCAGCCTGTTT \\
\hline >novel_mir_120 & 99 & TTTGTTCGTTCGGCTCGCGTGA \\
\hline >novel_mir_14 & 82 & CCGCACTGTGGGTACTTGCTGC \\
\hline >novel_mir_122 & 69 & TTCATTGTAGGAAGTTCAGGAGTCAC \\
\hline >novel_mir_5 & 69 & CAGTGCAATGTTAAAAGGGCATT \\
\hline >novel_mir_23 & 60 & TATTGCACTTGTCCCGGCCTGTT \\
\hline >novel_mir_133 & 52 & TAGGAACTTGTGCGGCAGGGATTT \\
\hline >novel_mir_112 & 50 & AAAGGAACATGTGGCAGCCAGGTG \\
\hline >novel_mir_22 & 49 & ACTGCAGTGAAGGCACTTGTAG \\
\hline >novel_mir_83 & 47 & TGGGTAAACTGATTAGCTGGCATC \\
\hline >novel_mir_64 & 42 & TACGTAGATATATATGTATTTT \\
\hline >novel_mir_76 & 42 & GCGGACGCGATGGCAGGCAGCAGG \\
\hline >novel_mir_41 & 41 & AGGAGTCTTGTTTCTACTTT \\
\hline >novel_mir_107 & 39 & TGAAGCAGAGCGCACGAACTCAA \\
\hline >novel_mir_101 & 38 & TGTAGTTGGAAGGATGCCCCTGGA \\
\hline >novel_mir_130 & 38 & TGGAAAACTGAGGACTGTCCGGCA \\
\hline >novel_mir_111 & 29 & TGGACAAGCTGGATTTCAAGT \\
\hline >novel_mir_121 & 25 & TACAAAATGGAGGAAGATCGGCAC \\
\hline >novel_mir_62 & 25 & CTGGGAGGTGGATGTTTACTT \\
\hline >novel_mir_126 & 23 & TGTGTGTAAGTGGGGAAGCTGG \\
\hline >novel_mir_66 & 23 & TTTGAAGACAAGCATAGCCTCATT \\
\hline >novel_mir_105 & 22 & TCTGGGTTAGACTGACGGCTTCG \\
\hline >novel_mir_71 & 21 & GCGCGCCGGCGTCCCGGGGGG \\
\hline >novel_mir_92 & 20 & TTCTAGTTGTGGCATATGGGAA \\
\hline >novel_mir_94 & 20 & TGCAGAGCACCGGCCACTGTGGGT \\
\hline >novel_mir_128 & 19 & CTGAGGAGATGTGGGAGAAGT \\
\hline >novel_mir_35 & 19 & ATGTCACTCGGCCCACTACCC \\
\hline >novel_mir_81 & 19 & AGGAAGCTGAGGCACATGGAAGTT \\
\hline >novel_mir_8 & 17 & CCGGGAGGCAGCGGGCGGGAA \\
\hline >novel_mir_87 & 17 & TCCAGGAAGGCGGGCACCAGGTG \\
\hline >novel_mir_118 & 16 & TGGCTGTTAGTTGAAATCCCGGTG \\
\hline >novel_mir_54 & 16 & TTGTTGGAGGGAATATACATAT \\
\hline >novel_mir_116 & 15 & AGGGTTTGTAGAGTGCAGCCGGC \\
\hline >novel_mir_70 & 15 & TGCAAGTAGGAAGACAGTGGGCTC \\
\hline >novel_mir_85 & 15 & TCGAACCTGTCAGGGCTGAGGCGG \\
\hline
\end{tabular}


Solexa Profiling Horse Testis miRNAs

\begin{tabular}{|c|c|c|}
\hline >novel_mir_88 & 15 & TGTAAAATGAGAGGGTTGGACTAA \\
\hline >novel_mir_119 & 13 & TGATTGTTCAGAGGGGATGAAGC \\
\hline >novel_mir_39 & 13 & CGGGGCAGCTCAGTACAGGATG \\
\hline$>$ novel_mir_58 & 13 & TAGGTAGTTTCATGTTGTTGGGA \\
\hline >novel_mir_95 & 13 & TAAGACGTATAGGCTGGTTCGGTA \\
\hline >novel_mir_108 & 12 & TGCAAAGCAGGAACGCTGGCCTC \\
\hline >novel_mir_11 & 12 & TGGAGGCAGCGAGGAGCACGT \\
\hline >novel_mir_131 & 12 & TGAATGTTGTGCAGCACTCCAA \\
\hline >novel_mir_16 & 12 & TCAGGCTCAGTCCCCTCCCGAT \\
\hline >novel_mir_91 & 12 & TGCCCCACAGGGAGATCCCGGA \\
\hline >novel_mir_134 & 11 & TCAGAGCAAGTAGTAATTCGAGA \\
\hline >novel_mir_29 & 11 & TCTTTGCAGGCTTGAACAAGGGTT \\
\hline >novel_mir_82 & 11 & TACTGTTGTGAGTGGATCGTCTC \\
\hline >novel_mir_93 & 11 & TCTCTTAGGGTGAAGTCTCGGGC \\
\hline >novel_mir_102 & 10 & TGCAGATGTCGGCACGAAGGATTT \\
\hline >novel_mir_103 & 10 & TGATAGACAGCGAGGAACCT \\
\hline >novel_mir_124 & 10 & TCCCATGAGATTCTGAGGCCAGGT \\
\hline >novel_mir_51 & 10 & AAATTACAGATTGTCTAAGAGG \\
\hline >novel_mir_80 & 10 & TCCAGGACTGTGGGGGCGCC \\
\hline >novel_mir_89 & 10 & TCCTAAGAGACTGAACAGAGGGA \\
\hline >novel_mir_98 & 10 & AGGGGCTGGGATTGGGGCAGGG \\
\hline Pnovel_mir_106 & 9 & TGGATGCAGTTTCTGGCAGAGCTC \\
\hline >novel_mir_125 & 9 & GTGGAGCTCTGGATCCAGGT \\
\hline >novel_mir_127 & 9 & TCTGATCACCCTGGGGCCCGACT \\
\hline >novel_mir_132 & 9 & AAGGGGTGAGGAAGACATAGTG \\
\hline >novel_mir_135 & 9 & TTCCTGTGGACATGGTGGGTGGG \\
\hline >novel_mir_69 & 9 & TCTCAGAAGCAGACCAAGGAATTC \\
\hline >novel_mir_78 & 9 & TGGGAAGCACCGGGGGACATCT \\
\hline >novel_mir_110 & 8 & TAGGGCTCGGTAACTAGGAGTGGA \\
\hline >novel_mir_113 & 8 & TTACAAGTAGGAGCATCTGTGTTT \\
\hline >novel_mir_20 & 8 & TATATATATATATGTACGTAT \\
\hline >novel_mir_75 & 8 & AGCAGATTTAGGGGATCAGGA \\
\hline >novel_mir_84 & 8 & TTACGTTCAAGCTCATCTCAGGTA \\
\hline >novel_mir_100 & 7 & AGCGTGGTTTGATGAGCAGG \\
\hline >novel_mir_114 & 7 & TAAGATGAGGAGAAAGGATTTGA \\
\hline >novel_mir_67 & 7 & TCGTGAAGTCGCTGGAGATGAGGC \\
\hline$>$ novel_mir_68 & 7 & TGAACTCAGAAGTAATTATGGACC \\
\hline >novel_mir_73 & 7 & TCCTAGGGGTTGAATGATTGGCT \\
\hline >novel_mir_74 & 7 & TCACAAGATGTTGGGATGGCGGGT \\
\hline >novel_mir_77 & 7 & TGGGGACGGCCTGGAGATAAAGG \\
\hline >novel_mir_86 & 7 & TGACATGGAGCAGCACGCCGAGGC \\
\hline >novel_mir_99 & 7 & TGTAAGTCAAGCTCTCTGAGGC \\
\hline >novel_mir_104 & 6 & TGAATGGACAGATGGATGGATG \\
\hline >novel_mir_109 & 6 & TGGATAAGGGGCAGAAAGAGTTTA \\
\hline
\end{tabular}

Braz. Arch. Biol. Technol. v.61: e18160122 2018 


\begin{tabular}{|l|l|l|}
\hline >novel_mir_129 & 6 & TGGAATAATCAGATGTGTAGGGGC \\
\hline >novel_mir_96 & 6 & TTGCAGTGATGACTTGAGTCTGT \\
\hline >novel_mir_115 & 5 & TGGCAGCTTGGATCACTTGAGTCT \\
\hline >novel_mir_117 & 5 & TGCTTGTCAGTGGTCTCAGGAAA \\
\hline >novel_mir_123 & 5 & CCGGAAGTAAGGCGTCTCTTCCTG \\
\hline >novel_mir_3 & 5 & ACCTTGGCTCTAGACTGCTTACT \\
\hline >novel_mir_34 & 5 & ATCATAGAGGAAAATCCACAT \\
\hline >novel_mir_43 & 5 & ACAGCTGTGAAATGGGCATGT \\
\hline >novel_mir_72 & 5 & TAACAGTCTCCAGTCACGGCCA \\
\hline >novel_mir_79 & 5 & TTGGCTCTGTGAGGTCGGCTCAA \\
\hline >novel_mir_90 & 5 & TGAGAGACGGTGGCAGGAAGTGT \\
\hline >novel_mir_97 & 5 & TGGGGTGGGGCTGGGGAGGGC \\
\hline >novel_mir_96 & 6 & TTGCAGTGATGACTTGAGTCTGT \\
\hline >novel_mir_115 & 5 & TGGCAGCTTGGATCACTTGAGTCT \\
\hline >novel_mir_117 & 5 & TGCTTGTCAGTGGTCTCAGGAAA \\
\hline >novel_mir_123 & 5 & CCGGAAGTAAGGCGTCTCTTCCTG \\
\hline >novel_mir_3 & 5 & ACCTTGGCTCTAGACTGCTTACT \\
\hline >novel_mir_34 & 5 & ATCATAGAGGAAAATCCACAT \\
\hline >novel_mir_43 & 5 & ACAGCTGTGAAATGGGCATGT \\
\hline >novel_mir_72 & 5 & TAACAGTCTCCAGTCACGGCCA \\
\hline >novel_mir_79 & 5 & TTGGCTCTGTGAGGTCGGCTCAA \\
\hline >novel_mir_90 & 5 & TGAGAGACGGTGGCAGGAAGTGT \\
\hline >novel_mir_97 & 5 & TGGGGTGGGGCTGGGGAGGGC \\
\hline
\end{tabular}

